\section{A Genetically Confirmed Intersubgeneric Hybrid in Nymphaea L. (Nymphaeaceae Salisb.)}

\author{
Donald H. Les ${ }^{1}$, Michael L. Moody, and Andrew S. Doran \\ Department of Ecology and Evolutionary Biology, University of Connecticut, \\ Storrs, CT 06269-3043
}

\author{
William E. Phillips \\ 3049 East Glengarry Road, Memphis, TN 38128
}

Additional index words. Anecphya, Brachyceras, Nymphaea gigantea, Nymphaea 'Andre Leu', Nymphaea colorata, nrITS, trnK 5' intron, cpDNA, molecular cloning, parsimony, water-lily

\begin{abstract}
A synthetic $\mathrm{F}_{1}$ water-lily hybrid has been obtained for the first time using parental species originating from different subgenera of Nymphaea. The cross was accomplished using Nymphaea gigantea Hook. 'Andre Leu' (subgenus Anecphya Casp.) as the maternal parent and a white-flowered variant of Nymphaea colorata Peter (subgenus Brachyceras Casp.) as the paternal parent. Morphologically, the hybrid possesses some characteristics of both parents, some intermediate features, and some unique traits. The cross was confirmed using DNA sequencing and molecular cloning techniques to compare biparentally inherited nuclear genetic markers in the parents and hybrid plant. Each parent possessed distinct alleles that were found to combine in the hybrid. Maternally inherited chloroplast DNA sequences confirmed $N$. gigantea as the maternal parent of the cross.
\end{abstract}

Since hybridization of water-lilies (Nymphaea) was initiated early in the 19 th century, claims of successful crosses have been challenged repeatedly due to poor documentation or to the lack of compelling corroborative evidence (Conard, 1905; Swindells, 1983). In 1804, George Spencer claimed to have produced a hybrid between Nymphaea dentata Schumach. \& Thonn. (=N. lotus L.) and $N$. rubra Roxb. ex Andrews (= Nymphaea pubescens Willd.) (Henkel et al., 1907); however, that report is undocumented otherwise. A similar cross between $N$. rubra and $N$. lotus (known as $N$. devoniensis Hook.) was reported in 1851 (Paxton, 1852), but that cross was doubted given that the alleged hybrid bore no resemblance to $N$. lotus morphologically, and native plants of $N$. rubra matching its description had been observed growing commonly in India (Conard, 1905; Perry, 1989). Plants exhibited in 1852 as $N$. ortgiesiana rubra (=N. 'Ortgiesiana-rubra') (Planchon, 1852) involved the same cross (N. ortgiesiana $=N$. lotus) but were given higher credibility because they were produced by dusting $N$. lotus pollen onto emasculated flowers of N. rubra and the resulting plants showed morphological traits intermediate between the parental taxa. To overcome such uncertainty, a search for more reliable methods of water-lily hybrid authentication continues to this day.

As for any aquatic plant, water-lily hybrids are difficult to document conclusively because

Received for publication 27 May 2003. Accepted for publication 23 Sept. 2003. We thank Michael Phillips and Virge Kask for technical and photographic assistance. Portions of this research were funded by NSF grant DEB-9806537.

${ }^{1}$ To whom reprint requests should be addressed. E-mail address: les@uconnvm.uconn.edu of extensive phenotypic plasticity, alternative causes of sterility and inadequate demonstration of hybrid criteria (Les and Philbrick, 1993). To exacerbate matters, many water-lily hybridizers became ever more secretive about their methods and parentages as new cultivars became lucrative financially (Conard, 1905; Swindells, 1983). Such practices heightened uncertainty regarding the pedigree of waterlilies touted as new hybrids (Conard, 1905), with many new cultivars suspected of being "... selected forms or improved seedlings of a species rather than a direct cross between two distinct parents" (Swindells, 1983).

Some plant breeders, such as George Pring (Pring, 1934a, 1934b), provided explicit data on crossing and selection regimes used in the development of water-lily hybrids. Yet, beyond the level of credibility provided by such welldocumented manipulations, the verification of putative water-lily hybrids has relied nearly exclusively on morphological criteria, i.e., the observation of different parental features in suspected hybrid plants (e.g., Pring, 1934a, 1934b). However, the application of morphology to detect hybrids is problematical because hybrid morphology can represent a combination of purely parental features, intermediate features, and extreme or novel features ( $\mathrm{Co}-$ nard, 1905; Rieseberg, 1995).

Despite numerous literature reports of $\mathrm{Nym}$ phaea hybrids (e.g., Conard, 1905; Henkel, 1907; Masters, 1974; Perry, 1989; Slocum et al., 1996; Swindells, 1983; Thomas, 1965), all known instances have involved species from within the same taxonomic subgenus as delimited by Conard (1905). Although a number of breeders claimed success with distant crosses involving species from different Nymphaea subgenera, Conard (1905) concluded that the reports were not credible and all lacked corroborating evidence. Conard remarked (1905; p. 221): "In spite of many attempts, no cross has yet succeeded between members of different subgenera of Nymphaea ...." Upon reviewing the record of unsuccessful hybridization attempts, Conard (1905) observed further: "It is notable that all of these failures involved two subgenera." More recently, Wiersema (1987) suggested that $N$. rudgeana G. Mey. possibly originated through intersubgeneric hybridization (subgenera Lotos DC. $\times \mathrm{Hy}$ drocallis (Planch.) Conard), but admitted that sufficient data were not available to confirm that possibility. Thus, up to this time, we have been unable to locate any reference providing conclusive evidence of any successful hybridization involving parents originating from different Nymphaea subgenera.

Padgett et al. $(1998,2002)$ reviewed criteria for evaluating natural plant hybrids in the water-lily genus Nuphar Sm. (Nymphaeaceae) based on recommendations made by Gottlieb (1972). In these studies, compelling evidence of hybridization was obtained using combined cytoplasmic (maternally inherited) and nuclear (biparentally inherited) molecular markers to demonstrate the genetic contributions of different parental genotypes in suspected hybrid plants. Genetic markers have become increasingly important as a means of overcoming interpretive problems associated with the complex expression of morphological traits in hybrids, as indicated by studies such as those of Rieseberg (1995).

Explicit documentation of $F_{1}$ hybrids has been achieved in the aquatic genus Myriophyllum L. (Haloragaceae) using a combination of molecular markers derived from the chloroplast and nuclear genomes (Moody and Les, 2002). That method targets a region of biparentally inherited, nuclear DNA in which the parents differ by a number of point mutations (Moody and Les, 2002). The $F_{1}$ hybrids between the parents display nucleotide polymorphisms at the variable sites. When individual copies of these nuclear sequences are isolated from the hybrids using molecular cloning techniques, they match either one or the other genotype otherwise unique to the parental species (Moody and Les, 2002). This approach confirms the contribution of alleles to the hybrid by each parent. In conjunction, the procurement of DNA sequences from a region of chloroplast DNA (cpDNA) that also differs in nucleotide composition between the parents, identifies the maternal contributor to the cross (by match) because of maternal inheritance of the marker (Moody and Les, 2002). This combined approach is effective at documenting hybrids because it directly verifies the presence of different alleles, each unique to a different species, combined within a single individual (i.e., a hybrid plant), while also indicating the specific direction of the cross by identifying the maternal parent of the hybrid.

In this study we use a similar approach to provide genetic confirmation of what we believe to be the first successful synthetic intersubgeneric hybrid in Nymphaea. This novel hybrid resulted from a cross using $N$. 
colorata (subgenus Brachyceras) as the pollen parent and a cultivar of $N$. gigantea (subgenus Anecphya) as the ovule parent.

\section{Materials and Methods}

Hybridization. Hybridization was performed using Nymphaea gigantea 'Andre Leu' (Anecphya) as the ovule parent and an unnamed, white-flowered variant of $N$. colorata (Brachyceras) as the pollen parent, both grown under greenhouse conditions. Stigmatic fluid from a first-day, early morning flower of $N$. 'Andre Leu' was removed using a medicine dropper and placed in a test tube. No ruptured anthers or dehisced pollen were visible in the newly opened bloom. The flower was not emasculated (or traumatized otherwise), as that method had proven unsuccessful in past hybridization attempts.

We anticipated that subsequent addition of Anecphya ( $N$. 'Andre Leu') pollen to a concentrated solution of Brachyceras $(N$. colorata) pollen (in Anecphya stigmatic fluid) might stimulate germination of the latter and possibly enhance pollen acceptance in the attempted intersubgeneric cross. Anthers from second-and third-day $N$. colorata flowers were removed with forceps and placed in the tube of Anecphya stigmatic fluid, which became yellow with pollen. Anthers were squeezed to remove excess stigmatic fluid, and the process was repeated three times. The Anecphya stigmatic fluid (now with a high concentration of Brachyceras pollen) was added back into the stigmatic cup of the $N$. 'Andre Leu' (Anecphya) plant while swirling to keep the dense pollen suspension from settling. The flower stem was labelled and left in this state for $4 \mathrm{~d}$, allowing self (Anecphya) pollen into the stigmatic fluid.

A nylon mesh bag was then stretched over the entire flower and tied around the peduncle, taking care to avoid unnecessary tension on the stalk. A 1-m piece of plastic tape was attached to the bag and then affixed to the side of the pond, allowing the developing ovary to submerge (for proper seed maturation), yet be retrieved later for examination of the enclosed contents. The hybridized $N$. 'Andre Leu' plant was illuminated for $16 \mathrm{~h}$ per day using a $1000-\mathrm{W}$, high-pressure sodium light situated at a height of $1.2 \mathrm{~m}$.

A plastic pan filled with clay to within 1 $\mathrm{cm}$ of the top was placed on a rack in a pond containing conditioned water at a temperature of $32^{\circ} \mathrm{C}$. Seeds were removed from the nylon bag, washed in pond water, and placed in a test tube containing pond water. The tube was inverted under water and the seeds were released to spread evenly across the clay substrate in the pan. A small piece of clay was placed in the water just above the seeds and rubbed gently to cover the seeds with a fine silt layer. The seeds and resulting seedlings were illuminated in the same manner as the hybridized plant (above).

Morphological markers. We compared states of 11 conspicuous morphological characters in the parental and hybrid plants (Table 1 ). Because only single plants bearing few flowers were involved, statistical evaluation of the quantitative morphological character states was not practical. However, for several quantitative traits (petal number, petal shape, filament width, number of leaf teeth/10 cm, tooth exsertion), a range of values from three separate observations was reported for comparison. Petal shape was approximated by the length : width ratio $(\mathrm{mm})$. Tooth exsertion was approximated using the ratio of tooth sinus width (mm) to the maximum tooth sinus depth (mm).

Molecular markers. We extracted total genomic DNA from frozen leaf material removed from the presumed pollen parent Nymphaea colorata [Phillips, s.n. (CONN)], the presumed ovule parent $N$. gigantea [Phillips, s.n. (CONN)], and their putative hybrid $N$. colorata $($ male $) \times N$. gigantea (female) [Phillips, s.n. (CONN)] using a modified CTAB extraction method (Doyle and Doyle, 1987). Voucher specimens of both parental plants and the hybrid are deposited at the Univ. of Connecticut herbarium (CONN). From total genomic DNA, we used PCR to amplify double-stranded DNA from the chloroplast $\operatorname{trnK}$ locus (including the $5^{\prime}$ intron flanking a portion of the matK coding region) and the nuclear ribosomal internally transcribed spacer (nrITS) region (including ITS-1, 5.8s rDNA, ITS-2 loci) following the methods described by Moody and Les (2002). Amplification of the ITS region used primers ITS4 and ITS5 as described by White et al. (1990); amplification of the cpDNA 5' trnK intron used the primers trnK-3914F and matK N-3-R as described by Les et al. (1999).

Automated methods employing an ABI 3100 DNA sequencer were used to obtain DNA sequences for the ITS region and for the 5'trnK intron from initial PCR-generated DNA samples (Moody and Les, 2002). The PCR products amplified from the ITS region were then cloned into plasmids using a TOPO TA cloning kit (Invitrogen, Carlsbad, Calif.). Ten clones from the putative hybrid and from each of the parental taxa were screened by sequencing (as above) the entire ITS region for all 30 clones.

The DNA sequences of $N$. colorata, $N$. gigantea, and the putative hybrid were aligned manually and analyzed for nucleotide polymorphisms and/or variable sites using the Sequencher 4.1.2 (Gene Codes Corp., Ann Arbor, Mich.) and MacClade 4 (Maddison and Maddison, 2000) software programs. We assembled a simplified data set of 11 sequences depicting the most commonly cloned ITS variants (two from N. colorata; two from $N$. gigantea; seven from the hybrid). The program PAUP 4.0b8 (Swofford, 2002) was used to assess the genetic relatedness of the DNA sequences by conducting a parsimony analysis using a heuristic search, random taxon addition, and tree bisection-reconnection, with all characters treated as unordered and weighted equally. Indels were treated as missing data. The degree of internal support for clades was evaluated by conducting 1000 bootstrap samples using the same search criteria described above for the parsimony analysis. The DNA sequences reported here have been deposited in the GenBank database under the following accession numbers: AY390327-AY390339.

\section{Results}

Hybridization. About 30 seeds were produced by the cross. Germination was low, with eight seedlings appearing over a 2 -week period. Only one seedling survived to maturity, which yielded the hybrid plant studied in this report (Fig. 1B; cover photo).
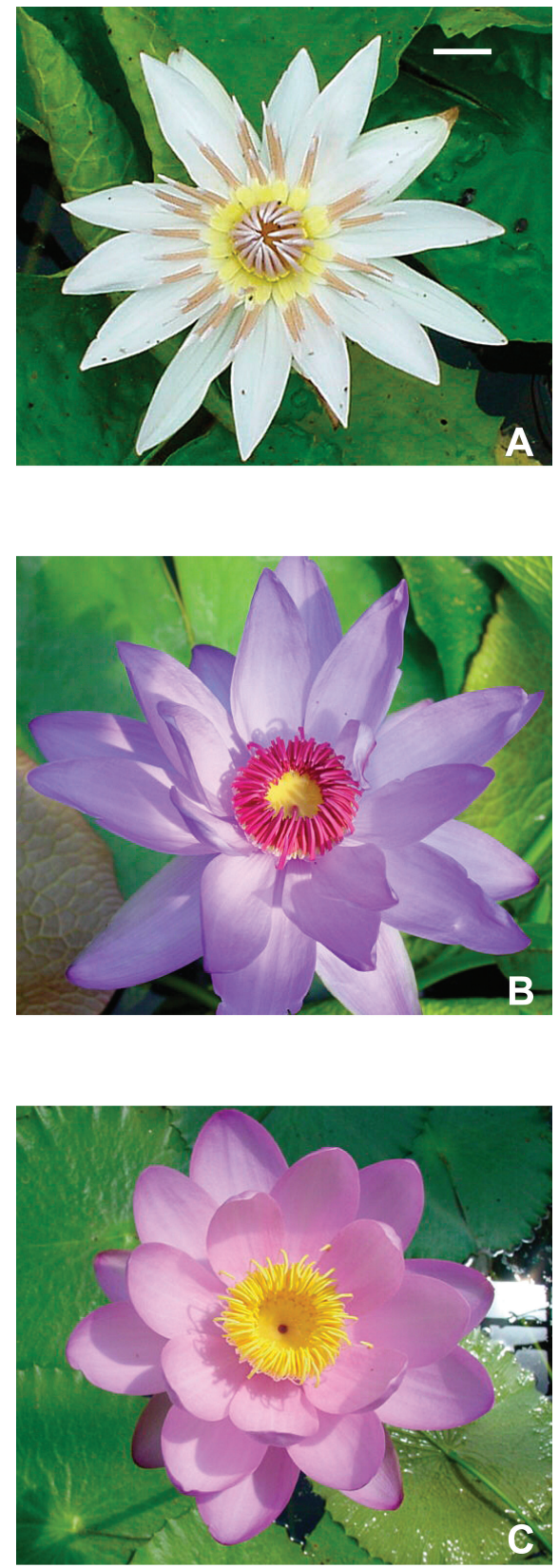

Fig. 1A-C. Water-lily (Nymphaea) species and their synthetic intersubgeneric hybrid: (A) white-flowered variant of Nymphaea colorata (subgenus Brachyceras) used as the pollen donor in an intersubgeneric cross; (B) a synthetic $F_{1}$ hybrid produced between species within different Nymphaea subgenera (A,C); (C) Nymphaea 'Andre Leu', a cultivar of Nymphaea gigantea (subgenus Anecphya) used as the maternal parent in an intersubgeneric cross. Scale bar (upper right) represents $\approx 2 \mathrm{~cm}$.

HortScience, Vol. 39(2), April 2004 
Table 1. Comparison of selected morphological features for Nymphaea colorata, $N$. gigantea 'Andre Leu', and their synthetic $\mathrm{F}_{1}$ hybrid. Symbols indicate hybrid traits that are: similar to $N$. colorata parent $(\mathrm{C})$; similar to $N$. gigantea parent $(\mathrm{G})$; intermediate to both parents (I); novel in the hybrid $(\mathrm{N})$; or inconclusive (unmarked).

\begin{tabular}{lllc}
\hline & $N$. colorata & $\mathrm{F}_{1}$ hybrid & N. gigantea \\
\hline Petal color (fresh) & white & violet $(\mathrm{N})$ & pink \\
Petal number & $13-14$ & $14-16$ & $12-17$ \\
Petal shape (length : width) & $3.5-4.3$ & $2.3-3.3(\mathrm{I})$ & $2.2-3.0$ \\
Petaloid sepals & present & present $(\mathrm{C})$ & absent \\
Stamen number (approximate) & 80 & $>200(\mathrm{G})$ & $>200$ \\
Stamen filament width (mm) & $1.5-4.0$ & $0.5-2.5(\mathrm{I})$ & $0.2-0.3$ \\
Anther color (fresh) & white & carmine $(\mathrm{N})$ & yellow \\
Projecting floral axis color & yellow & carmine $(\mathrm{G})$ & carmine \\
Leaf tooth exsertion (width : depth) & $3.4-5.6$ & $1.5-2.7(\mathrm{G})$ & $2.3-2.8$ \\
Number of leaf teeth/10 cm & $1-6$ & $5-7(\mathrm{I})$ & $7-8$ \\
Major leaf vein number & 17 & $17-19(\mathrm{C})$ & 14 \\
\hline
\end{tabular}

\section{subg. Anecphya}

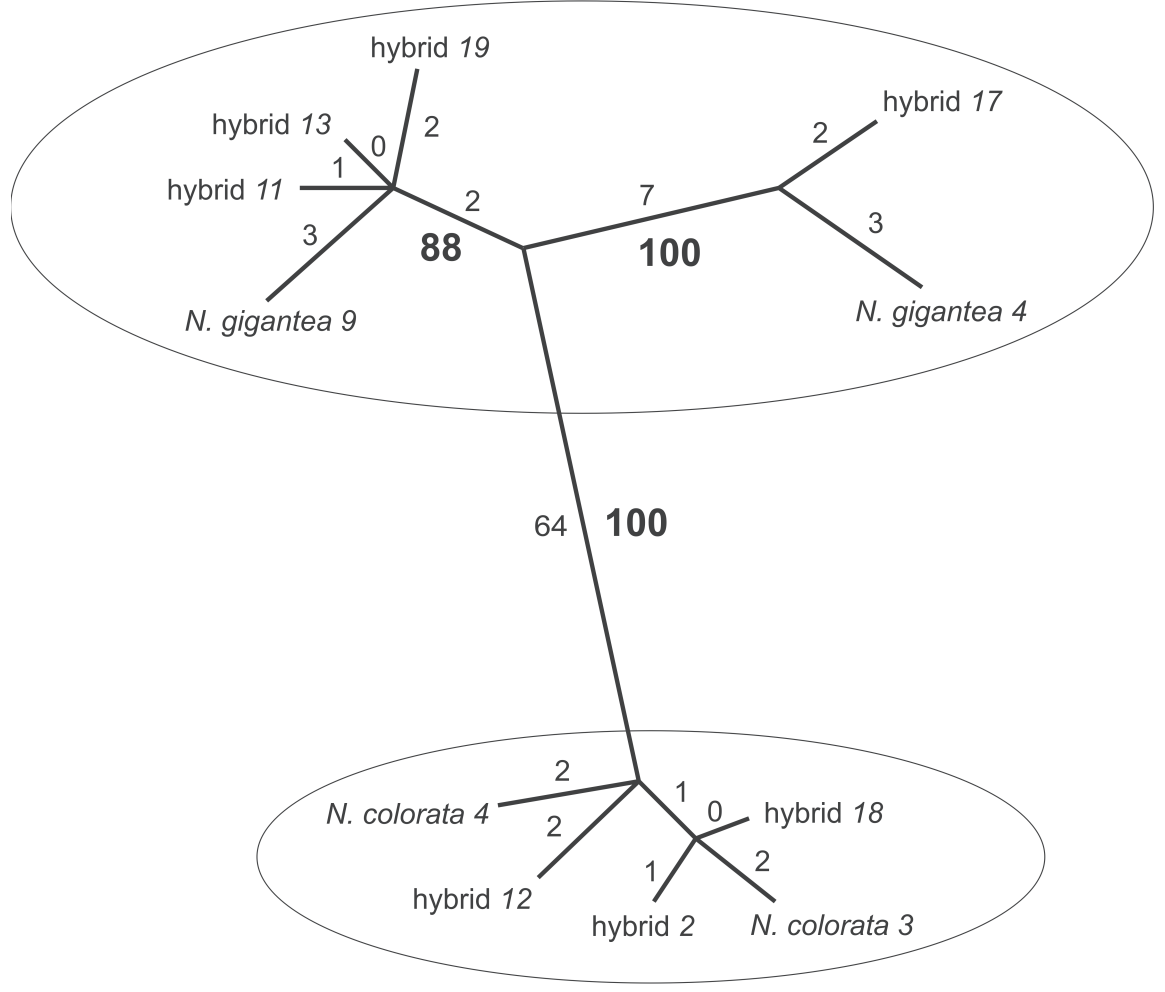

subg. Brachyceras

Fig. 2. Strict consensus of the four most parsimonious trees of 92 steps $(C I=1.00)$ obtained from analysis of ITS sequences cloned from two water-lily species and their synthetic $F_{1}$ hybrid. The upper clade represents allelic sequences matching Nymphaea gigantea (subgenus Anecphya) and the lower clade those of $N$. colorata (subgenus Brachyceras). Italicized numbers following names refer to clone numbers. Other numbers not in bold type are branch lengths; numbers in bold type are bootstrap values. This tree demonstrates that some ITS alleles cloned from the $\mathrm{F}_{1}$ hybrid plant match those otherwise exclusive to one parent and some match those otherwise exclusive to the other parent used in the cross.

Morphological markers. For the 11 morphological characteristics that we examined (Table 1), the putative hybrid plants possessed two traits (violet petal color; carmine anther color) different from either parent, three traits (petal shape; filament width; number of leaf teeth) intermediate to $N$. colorata and $N$. gigantea, two traits (petaloid sepals; higher major leaf vein number) shared with $N$. colorata, and three traits (projecting floral-axis color; higher stamen number; lower leaf-tooth exsertion) shared with $N$. gigantea. Petal number was inconclusive due to overlap between the parental species. trees. Two $N$. gigantea clades were well-supported, with nine point mutations varying between these two clades. There was one wellsupported N. colorata clade. All 10 clones of $N$. colorata fell within one clade, with all 10 clones of $N$. gigantea falling within a different clade; the 10 clones originating from the hybrid fell either within the clade representing $N$. colorata or that of $N$. gigantea. Each clade contained representative sequences cloned from the alleged hybrid taxon (Fig. 2). The occurrence in the hybrid of allelic nuclear DNA sequences different from those otherwise fixed exclusively in either $N$. colorata or $N$. gigantea confirmed that hybridization between these parents had occurred.

The cpDNA (trnK $5^{\prime}$ intron) sequences of $N$. gigante $a$ and the hybrid were identical, with both differing from $N$. colorata by two point mutations. The match of the cpDNA sequences confirmed $N$. gigantea as the maternal parent of the hybrid.

\section{Discussion}

With introductions of new Nymphaea cultivars occurring continuously, there is an increasing need to reinforce traditional horticultural taxonomy by providing more compelling evidence of hybridization than can be obtained by the "expert eye" (Grant and Culham, 1997). In particular, the augmentation of morphological data with molecular markers has become the method of choice for studying relationships of cultivated plants (Andrews et al., 1999).

Traditionally, the generation of hybrid water-lily cultivars has relied on a relatively simple method of documentation. Viable seed production is the first indication that a presumed cross has been successful. Adult plants raised from the alleged hybrid seed are then evaluated phenotypically in search of morphological qualities characteristic of both assumed parents. Plants possessing such a mix of characters are concluded to be of hybrid origin. Although this approach is relatively straightforward, problems can arise that compromise the acceptance of such conclusions.

The methods practiced by hybridizers vary extensively. Ideally, flowers are bagged and emasculated to preclude foreign pollen contamination. However, this method is not always appropriate. In the case of the intersubgeneric water-lily hybrid, we found that floral emasculation deterred crossing success, as evidenced by repeated failures using that approach. We believe that keeping stamens intact on the maternal parent reduces stress on the flower and the presence of self pollen may stimulate germination of foreign pollen in the stigmatic fluid and enhance its success. Thus, in such instances where absolute exclusion of foreign pollen is difficult or impossible to achieve, it can be challenging to demonstrate conclusively whether a plant represents a particular hybrid or has resulted instead from contamination by self pollen or pollen from a source other than assumed. Conventionally, this question is resolved by phenotypic evaluation as described above.

However, phenotypic evaluations are inher- 
ently fallible. As Conard (1905) emphasized, variation in water-lilies follows no fixed pattern of inheritance, with hybrid offspring exhibiting arrays of intermediate, dominant, or exaggerated parental characters. Furthermore, natural variation and novel genetic recombination can lead to unique phenotypes within a species quite independently of hybridization (Conard, 1905). Clearly, there are limitations to the sole use of morphological criteria for confirming water-lily hybrids and, as a result, some level of doubt will remain in many cases.

The use of genetic markers overcomes these limitations by providing an unambiguous means of demonstrating whether hybridization has occurred by assigning parentage in a virtually unequivocal fashion. Parental plants need only to differ exclusively for a genetic marker that is inherited biparentally, i.e., one originating from the nuclear genome. Once unique parental markers are identified, evidence of hybridization is provided by the recovery of both parental markers from the same individual plant. Similarly, maternally inherited markers (e.g., most plant cpDNA sequences) will effectively identify the maternal parent of the cross.

The intersubgeneric hybrid water-lily reported here represents a case where genetic confirmation is necessary. First, this hybrid represents a cross that has been attempted for more than a century, but hitherto unsuccessfully. Accordingly, any claim of success should be accompanied by evidence of the most definitive nature. Furthermore, because the hybridization method did not incorporate floral emasculation, contamination by self pollen was possible and had to be ruled out.

In itself, the morphological evidence of this intersubgeneric cross was consistent with hybridization but not conclusive. The putative hybrid plant exhibited some features intermediate to both parents and some that resembled either Nymphaea colorata or N. gigantea (Table 1). However, a few characteristics (notably with respect to floral coloration patterns) were singularly unique. If the progeny simply resulted from selfing of $N$. gigantea (the maternal parent used in the cross), then one would not expect to observe phenotypic characteristics of $N$. colorata. However, one characteristic (larger number of major leaf veins) is variable in the hybrid and provides relatively weak evidence of genetic contributions from $N$. colorata. Better evidence is provided by the presence of petaloid sepals and stamen filament width. Filaments are always narrowly filiform (never laminar) in $N$. gigantea and always laminar in $N$. colorata. The presence in the putative hybrid of petaloid sepals and intermediate filaments, some relatively laminar in shape, provides more compelling evidence that some genetic contribution from $N$. colorata had indeed occurred.

Molecular markers provided definitive evidence of intersubgeneric hybridization. First, the two parental species showed extensive divergence in the nrITS region, which differed by 64 diagnostic nucleotide substitutions (Fig. 2). Such a high level of nucleotide divergence is consistent with the taxonomic placement of these taxa within different subgenera as suggested by Conard (1905). Slight divergence was observed among sequences cloned from each parental species. ITS sequences cloned from $N$. colorata showed several variants, each differing by a few (1-4) substitutions, but all representing one distinct allelic type (Fig. 2). Minor sequence variation of this extent (e.g., Moody and Les, 2002) results as an artifact of the amplification of cloned PCR products (Invitrogen, pers. comm.). The ITS sequences cloned from $N$. gigantea showed similar minor levels of variation. However, we also observed additional variation that was not simply random, but was shared by a number of sequence variants, thus indicating the presence of several distinct ITS sequences in that species (Fig. 2). These different ITS allelic variants within $N$. gigantea may represent paralogous sequences attributable to duplications of the ITS region (possibly due to polyploidy). Evidence that the minor sequence variants had resulted as cloning artifacts was indicated by the initial ITS sequences obtained prior to cloning. The ITS sequence chromatograms of both parents were relatively "clean," i.e., showing little evidence of polymorphisms manifest as multiple signal peaks. However, $N$. gigantea did exhibit polymorphisms at the sites that differed consistently between its own sequence variants. Moreover, despite the relatively minor level of intraspecific sequence variation encountered in ITS, none of the $N$. colorata clones examined showed any of the $N$. gigantea allelic variants, nor did any of the $N$. gigantea clones exhibit any of the $N$. colorata allelic variants as is demonstrated by the perfect consistency index (CI) of 1.00. Thus, these two widely divergent ITS allelic groups can be regarded as reliable genetic markers specific to each of the plants used as parents in the hybridization.

Genetic evidence of hybridization in the putative hybrid plant was manifest variously. First, the ITS sequence chromatograms obtained initially from the putative hybrid plant (prior to cloning) exhibited obvious polymorphisms at the sites differentiating the parental species. These polymorphic sites indicated the presence of both nucleotides that otherwise were exclusive to only one of the parental sequences. Subsequent cloning and sequencing of single copies from these mixed pools of alleles showed conclusively that the putative hybrid plant contained allelic variants matching those of both parental plants (Fig. 2), thereby providing compelling genetic evidence of hybridization. Furthermore, the exact match of the maternally inherited $\operatorname{trnK}$ 5 ' intron sequence, between the alleged hybrid plant and $N$. gigantea, implicated this species as the maternal parent of the cross, thus also in agreement with the directionality followed in the hybridization methods.

The combination of additive and intermediate morphology and additive genetic markers specific to the two divergent parents used in the hybridization is taken to provide compelling evidence that intersubgeneric hybridization now has been achieved successfully in the genus Nymphaea. The new hybrid is described in a companion article (Doran et al., 2004).

\section{Literature Cited}

Andrews, S., A. Leslie, and C. Alexander. 1999. Taxonomy of cultivated plants. Royal Bot. Gardens, Kew.

Conard, H.S. 1905. The waterlilies: A monograph of the genus Nymphaea. Carnegie Inst., Washington, D.C.

Doran,A.S., D.H. Les, M.L. Moody, and W.E. Phillips. 2004. Nymphaea 'William Phillips', a new intersubgeneric hybrid. HortScience 39:446-447.

Doyle, J.J. and J.L. Doyle. 1987. A rapid DNA isolation procedure for small quantities of fresh leaf tissue. Phytochem. Bul. 19:11-15.

Gottlieb, L.D. 1972. Levels of confidence in the analysis of hybridization in plants. Ann. Missouri Bot. Gard. 59:435-446.

Grant, M. and A. Culham. 1997. DNA fingerprinting and the identification of cultivars. The New Plantsman 4:85-87.

Henkel, F., F. Rehnelt, and L. Dittman. 1907. Das Buch der Nymphaeaceen oder Seerosengewächse. Friedrich Henkel, Darmstadt.

Les, D.H. and C.T. Philbrick. 1993. Studies of hybridization and chromosome number variation in aquatic plants: Evolutionary implications. Aquat. Bot. 44:181-228.

Les, D.H., E.L. Schneider, D.J. Padgett, P.S. Soltis, D.E. Soltis, and M.Zanis. 1999. Phylogeny, classification and floral evolution of water lilies (Nymphaeales): A synthesis of non-molecular, rbcL, matK and 18S rDNA data. Syst. Bot. 24:28-46.

Maddison, D.R. and W.P. Maddison. 2000. MacClade 4: Analysis of phylogeny and character evolution. Sinauer Assoc., Sunderland, Mass.

Masters, C.O. 1974. Encyclopedia of the water-lily. TFH Publ., Neptune City, N.J.

Moody, M.L. and D.H. Les. 2002. Evidence of hybridity in invasive watermilfoil (Myriophyllum) populations. Proc. Natl. Acad. Sci. USA 99:14867-14871.

Padgett, D.J., D.H. Les, and G.E. Crow. 1998. Evidence for the hybrid origin of Nuphar $\times$ rubrodisca (Nymphaeaceae). Amer. J. Bot. 85:1468-1476.

Padgett, D.J., M. Shimoda, L.A. Horky, and D.H. Les. 2002. Natural hybridization and the imperiled Nuphar (Nymphaeaceae) of western Japan. Aquat. Bot. 72:159-172.

Paxton. J. 1852. N. devoniensis. Gardener's Chronicle, 10 July 1852.

Perry, F. 1989. Waterlilies and other aquatic plants. Holt, New York.

Planchon, J.E. 1852. N. Ortgiesiano-rubra. Flore des Serres 8:67-73; tab. 775-6. Ghent, 1852-53.

Pring, G.H. 1934a. Hybrid Nymphaeas. Missouri Bot. Gard. Bul. 22:47-90.

Pring, G.H. 1934b. Hybrid Nymphaeas - Their creation, propagation, and cultivation. Missouri Bot. Gard. Bul. 22:93-108.

Rieseberg, L.H. 1995. The role of hybridization in evolution: Old wine in new skins. Amer. J. Bot. 82:944-953.

Slocum, P.D., P. Robinson, and F. Perry. 1996. Water gardening: Water lilies and lotuses. Timber Press, Portland, Ore.

Swindells, P. 1983. Waterlilies. Timber Press, Portland, Ore.

Swofford, D.L. 1998. PAUP*: Phylogenetic analysis using parsimony. Vers. 4.0. Sinauer Assoc., Sunderland, Mass.

Thomas, G.L. 1965. Goldfish pools water-lilies and tropical fishes. H-919. TFH Publ., Neptune City, N.J.

White, T.J., T. Bruns, S. Lee, and J. Taylor. 1990. Amplification and direct sequencing of fungal ribosomal RNA genes for phylogenetics, p. 315-322. In: M.A. Innis, D.H. Gelfand, J.J. Sninsky, and T.J. White (eds.). PCR protocols: A guide to methods and applications. Acad.Press, San Diego.

Wiersema, J.H. 1987. A monograph of Nymphaea subgenus Hydrocallis (Nymphaeaceae). Vol. 16, Systematic botany monographs. Amer. Assn. Plant Taxonomists. 